Communication, technologies et développement

$6 \mid 2018$

Technologies mobiles, innovation et développement

\title{
Laurence Devillers, Des robots et des hommes, mythes, fantasmes et réalité
}

Éditions Plon, Paris, 2017, 237 pages

\section{Cécile Dolbeau-Bandin}

\section{CpenEdition}

\section{Journals}

Édition électronique

URL : http://journals.openedition.org/ctd/917

DOI : $10.4000 /$ ctd. 917

ISSN : 2491-1437

Éditeur

Chaire Unesco Pratiques émergentes en technologies et communication pour le développement

Référence électronique

Cécile Dolbeau-Bandin, "Laurence Devillers, Des robots et des hommes, mythes, fantasmes et réalité », Communication, technologies et développement [En ligne], 6 | 2018, mis en ligne le 18 décembre 2018 consulté le 23 septembre 2020. URL : http://journals.openedition.org/ctd/917 ; DOI : https://doi.org/ $10.4000 /$ ctd. 917

Ce document a été généré automatiquement le 23 septembre 2020

Communication, technologies et développement 


\section{Laurence Devillers, Des robots et des hommes, mythes, fantasmes et réalité}

Éditions Plon, Paris, 2017, 237 pages

Cécile Dolbeau-Bandin

\section{RÉFÉRENCE}

Laurence Devillers, Des robots et des hommes, mythes, fantasmes et réalité, Éditions Plon, Paris, 2017, 237 pages

1 L'ouvrage s'adresse à un large public de ce qu'on peut et qu'on pourra entreprendre avec des robots sociaux. Laurence Devillers (professeure à l'Université Paris-Sorbonne 4 en informatique et spécialiste des interactions entre homme-machine et de la robotique sociale) s'interroge sur le rôle des robots sociaux qui nous aideront demain à gérer notre quotidien. Il s'agit d'expliquer qu'il ne faut pas en avoir peur et qu'il faut apprendre à les connaître. Il faut se préparer à vivre avec ceux qui deviendront nos assistants, nos aides, nos compagnons de tous les instants. Il faut apprivoiser ces robots sociaux qui devront « respecter nos valeurs humaines pour notre bien-être » (p. 43).

L'ouvrage commence par une fiction, c'est une mise en abyme. Ce procédé littéraire consiste à placer à l'intérieur du texte principal un texte qui reprend les thèmes du texte principal. En 2025, cinq robots humanoïdes (LILY, THEO, AIKO, RO42 et JEDI) de la série Marvin sont livrés dans un immeuble parisien de la société franco-japonaise ROBOTA à cinq usagers différents. Ce sont des robots conversationnels affectifs pour assister ces personnes à leurs domiciles (éviter l'isolement, prévenir, les chutes, surveiller leur santé....). Il est possible de choisir son niveau d'adaptation à l'humain (le robot n'apprend pas ou il peut apprendre ou il peut devenir un compagnon en s'adaptant aux habitudes des humains, p. 13) et de le personnaliser (voix, couleur des yeux...). Ainsi, « la machine va s'adapter à l'humain et l'humain à la machine » (p. 43). Ces cinq robots sociaux ne sont pas encore opérationnels en 2017 : l'intelligence des robots c'est-à-dire son principe d'apprentissage est lié à l'imitation et à l'association 
pour répondre à des missions spécifiques. En revanche, ils seront bientôt prêts et cette fiction permet de se projeter dans un avenir proche avec des robots.

3 L'avant-propos présente ce que sont les principaux robots d'aujourd'hui (PARO, NAO, PEPPER, ASIMO, les agents conversationnels ou chatbots...) et ce qu'ils peuvent apporter de positif (effet thérapeutique auprès d'enfants autistes...). Ce sont des robots assistants qui ne remplacent pas les humains. Par exemple dans la santé, ils pourraient assister des personnes dépendantes pour rester plus longtemps chez elles. Les scientifiques doivent et devront travailler de manière pluridisciplinaire en expliquant leur travail et les enjeux des projets qu'ils développent ou observent.

La première partie fait référence à de nombreux récits comme M. Shelley, I. Asimov, K. Capek, J. Mac Carthy ; films comme Metropolis, 2001, l'odyssée de l'espace, Stars wars, Her, Terminator, Wall-E, Ex Machina...; séries télévisées comme Westworld, Real Humans; mangas comme Astroboy ; des animations come Goldorak, Ulysse 31... Les dérives ou les risques de l'intelligence artificielle (IA) sont en général au coeur de l'intrigue de ces différentes œuvres. Ces premières idées de la créature artificielle sont issues la mythologie grecque avec la statue Galatée et de la religion avec le Golem. Ici, la science fiction précède la science. La robotique est marquée par les trois lois et la loi zéro d'I. Asimov et qui sont les premiers socles de «l'idée d'une machine morale» (p. 50). L'œuvre d'I. Asimov est bien « une projection dans le futur de l'acceptabilité éthique et sociale des robots intelligents » (p. 49). Quittant la science-fiction, cette face sombre de la robotique est reprise par les «transhumanistes». Ce mouvement promet l'éternelle jeunesse et veut augmenter les capacités de l'homme, le faire vivre plus de 500 ans voire le rendre immortel grâce au développement de l'intelligence artificielle.

Présent dans notre quotidien, le secteur de l'IA est en pleine expansion grâce aux investissements des GAFA (Google, Apple, Facebook et Amazon) et du BATX chinois (Baidu, Alibaba, Tencent et Xiaomi). Mais l'IA est un concept polysémique difficile à définir et à concevoir. Un petit nombre d'entrepreneurs et scientifiques (E. Musk, S. Hawskins...) mettent en garde contre les dérives de l'IA. La grande majorité des experts en IA sont cependant plus prudents sur les performances des systèmes d'intelligence artificielle : "les « robots égaux ou supérieurs aux hommes ne sont pas pour demain » (p.106). Les approches symboliques et des raisonnements logiques dans les années 1960 s'accompagnent de trois hivers (ralentissement des recherches en IA à cause des promesses non tenues) de l'IA (1974 à 1980, de 1987 à 1993, 1980-1990). Mais aujourd'hui, l'IA change : elle passe de l'IA conçue pour une mission précise (jouer aux échecs) pour passer à une IA plus générale capable de s'adapter à plusieurs missions et prendre des décisions de façon autonome. Une IA qui apprend (deep learning) est prometteuse mais elle interpelle sur ses risques et enjeux éthiques (surtout si elle reste dans les mains des cinq géants du web).

La deuxième partie présente les robots aux formes différentes (humanoïde...) et aux usages différents (industriel, de service). Les robots sont des machines capables d'« imiter les hommes sur des tâches spécifiques » (p. 114) qui interagissent avec son environnement. Cette machine artificielle intelligente, avec des modèles informatiques spécifiques, perçoit, se déplace, peut apprendre, interagit.... La robotique industrielle repose sur les $4 \mathrm{D}$ « Dangerous, Dull, Dirty, Dumb » et la robotique sociale sur les $4 \mathrm{E}$ «Everyday, E-health, Education, Enternainment». Un robot social est un assistant ou compagnon qui détectera nos émotions pour y répondre de façon adéquate. Les chercheurs explorent toutes les possibilités qu'offrent ou vont offrir ces robots sociaux. 
Certains imaginent même d'utiliser des robots sexuels pour soigner les pathologies sexuelles les plus graves.

7 Un robot social n'a pas d'empathie artificielle: mon robot simule quand il me dit je t'aime. Il n'est pas capable de ressentir. Pour que nous l'acceptions, le robot devra être capable de communiquer et de s'adapter à nous, les robots sociaux sont aptes à nous envoyer un feedback émotionnel. Ainsi, ils identifient les émotions d'un humain en se basant sur des indices physiques (mimiques, posture...). Une fois les réactions identifiées, ces robots y répondent avec des expressions et gestuelles adaptées; et entretiennent l'illusion de comprendre les états affectifs de l'homme et d'y être sensibles. Le robot est et restera une machine à simuler. Seulement, elle le fera de mieux en mieux et nous serons plusieurs à croire que cet amour est réel et bien réciproque.

Le défi est de créer une machine suffisamment intelligente pour avoir une conscience morale, ou au moins un premier niveau de conscience de soi. Il faut comprendre ce qu'elle sait faire, le traitement de l'usage des données, des accès néfastes (piratage) et la triade «homme-robot-médecin/famille». Il n'est pas question de faire des robotssubstituts à la présence humaine. La robotique sociale s'accompagnera d'une multiplicité d'usages qui pourront mettre en question les normes juridiques et provoquer des questionnements moraux ou éthiques. Ce qui nécessite de mener quelques réflexions et de prendre quelques précautions dès la conception des recherches. Il fait éduquer, rendre compte, comprendre et analyser les peurs et fantasmes liés à la robotique (piratage, super-intelligence artificielle, surveillance en continu, usages des robots comme armes, deshumanisation, clivage dans la société, remplacement des hommes). Communication, compréhension, éducation et pédagogie sont les maitres-mots de cette éthique de la robotique sociale : il faut définir tous en ensemble sa fonction et savoir l'utiliser pour bien vivre cette prochaine " coévolution humain-robot ».

L'épilogue prolonge les trois lois et la loi zéro de la robotique d'I. Asimov en proposant onze commandements pour construire des robots sociaux avec « des comportements éthiques et sécuritaires" (p. 228). Ces commandements ouvrent une réflexion collective sur la robotique sociale et l'intelligence artificielle, indispensable pour notre futur vivre-ensemble humain-robot.

10 C'est un ouvrage indispensable et complet sur la robotique sociale. Il nous aide à prendre du recul et à entrevoir une intelligence artificielle maîtrisée (sécurité de la robotique), utile (aide ou assistance) et démystifiée (visons iréniques sans fondement). Seulement, l'attachement des humains envers les objets n'est pas assez développé (Tisseron, 2016). Cette référence expliquerait parfaitement notre attachement futur aux robots sociaux. Il est aussi dommage que ce texte n'est pas mentionné l'apport des sociologues et de spécialistes en Sciences de l'information de la communication à la robotique sociale : la formation de Véronique Aubergé en informatique, en traitement du signal, en sciences de la communication et en sciences du langage illustre parfaitement cette merveilleuse et nécessaire complémentarité pour observer et comprendre la robotique sociale d'aujourd'hui et de demain. 


\section{AUTEURS}

\section{CÉCILE DOLBEAU-BANDIN}

Université de Caen 